\title{
Herméneutique et sciences de l'information : un état de la question
}

\author{
Hermeneutics and Information Science: A Review of the \\ Situation
}

\section{Hermenéutica y ciencias de la información: situación actual}

\section{Daniel Ducharme}

Volume 46, numéro 1, janvier-mars 2000

URI : https://id.erudit.org/iderudit/1032682ar

DOI : https://doi.org/10.7202/1032682ar

Aller au sommaire du numéro

Éditeur(s)

Association pour l'avancement des sciences et des techniques de la documentation (ASTED)

ISSN

0315-2340 (imprimé)

2291-8949 (numérique)

Découvrir la revue

Citer cet article

Ducharme, D. (2000). Herméneutique et sciences de l'information : un état de la question. Documentation et bibliothèques, 46(1), 15-25.

https://doi.org/10.7202/1032682ar
Résumé de l'article

Cet article porte sur les applications possibles de la philosophie herméneutique aux sciences de l'information. Après avoir justifié le recours à l'herméneutique par des arguments d'ordre épistémologique, l'auteur passe en revue les champs d'application qui se résument à l'analyse documentaire, la communication de l'information (référence), la gestion des documents structurés en hypertexte ainsi qu'à l'évaluation des archives et détermine, ce faisant, quelle herméneutique les auteurs intègrent dans leurs recherches. En conclusion, l'auteur évalue la pertinence d'appliquer cette philosophie aux domaines des sciences de l'information.
Tous droits réservés (C) Association pour l'avancement des sciences et des techniques de la documentation (ASTED), 2000
Ce document est protégé par la loi sur le droit d'auteur. L'utilisation des services d’Érudit (y compris la reproduction) est assujettie à sa politique d'utilisation que vous pouvez consulter en ligne.

https://apropos.erudit.org/fr/usagers/politique-dutilisation/ 


\title{
Herméneutique et sciences de l'information: un état de la question
}

\author{
Daniel Ducharme \\ Archiviste \\ Archives nationales du Québec
}

Cet article porte sur les applications possibles de la philosophie herméneutique aux sciences de l'information. Après avoir justifié le recours à l'herméneutique par des arguments d'ordre épistémologique, l'auteur passe en revue les champs d'application qui se résument à l'analyse documentaire, la communication de l'information (référence), la gestion des documents structurés en hypertexte ainsi qu'à l'évaluation des archives et détermine, ce faisant, quelle herméneutique les auteurs intègrent dans leurs recherches. En conclusion, l'auteur évalue la pertinence d'appliquer cette philosophie aux domaines des sciences de l'information.

\begin{abstract}
Hermeneutics and Information Science: $A$ Review of the Situation

This article describes the possible applications of hermeneutics to the information sciences. Following a justification of hermeneutics based on epistomological arguments, the author reviews the following applications: document analysis, the communication of information (reference), hypertext record management and archival assessment. Such a review allows the author to identify what hermeneutics various authors incorporate into their research. In concluding, the author evaluates the pertinence of applying this philosophy to the areas of information science.
\end{abstract}

Hermenéutica y ciencias de la información: situación actual Este artículo trata sobre las posibles aplicaciones de la filosofía hermenéutica a las ciencias de la información. Después de justificar el uso de la hermenéutica con argumentos epistemológicos, el autor examina los campos de aplicación, que se reducen al análisis de documentos, la comunicación de la información (referencias), la gestión de documentos estructurados en hipertextos y la evaluación de los archivos; $y$, de este modo, determina la hermenéutica que los autores integran en sus investigaciones. En conclusión, el autor evalúa la pertinencia de aplicar esta filosofía a los campos de las ciencias de la información.
Cet article porte sur les applications possibles de la philosophie herméneutique aux sciences de l'information 1 . Par sciences de l'information, nous entendons ce champ spécifique du savoir qui a pris pour objet d'étude l'information véhiculée sous forme de «documents », c'est-à-dire à travers différents supports d'information: documents de bibliothèques ou de centres de documentation, documents d'archives, documents informatiques, documents Web, etc. D'origine anglo-saxonne, ce regroupement de sciences pénètre peu à peu le monde francophone et, à cet égard, l'École de bibliothéconomie et des sciences de l'information de l'Université de Montréal, de par la notoriété et la diffusion de ses recherches, constitue une pionnière dont l'exemple est suivi par plusieurs pays de la francophonie.

L'objectif de cet article consiste essentiellement à recenser les écrits relatifs à I'herméneutique en sciences de l'information et à déterminer leurs champs d'application. Après avoir défini les sciences de l'information et, dans une moindre mesure, l'herméneutique, nous tenterons de comprendre, dans un premier temps, pour- quoi les auteurs en sciences de l'information s'intéressent à la théorie de l'interprétation. Pour ce faire, nous résumerons les arguments invoqués par ces derniers pour justifier l'application de l'herméneutique aux sciences de l'information. Puis, dans un deuxième temps, nous passerons en revue les domaines d'application retenus par les auteurs. Outre les considérations de nature générale, ces domaines se résument essentiellement à l'analyse documentaire (bibliographie), à la référence (communication de l'information), à la gestion des documents structurés en hypertexte et à l'évaluation des archives. Quelle herméneutique les auteurs en sciences de l'information intègrent-ils dans leurs recherches? C'est ce que nous tenterons de déterminer. Nous verrons alors que les philosophes consultés par les auteurs Ricoeur, Heidegger, Gadamer, Betti, Derrida, etc. - relèvent de plusieurs écoles de pensée. Enfin, en conclusion, nous évaluerons la pertinence d'appliquer l'herméneutique aux domaines des sciences de l'information.

Cette modeste recherche provient d'un archiviste, donc d'une personne étrangère au milieu de la philosophie. Cela apporte sans doute une coloration particulière au développement, essentiellement pratique, de ce document. D'aucuns diront que nous avons tort de chercher des applications concrètes à une pensée orientée surtout vers la compréhension du comprendre. II n'en demeure pas moins que, en sciences de l'information, des auteurs prétendent appliquer l'herméneutique à des situations qui répondent à des préoccupations professionnelles. Reste à savoir s'il s'avère pertinent de le faire et, si oui, dans quelle mesure. C'est justement le but que nous nous proposons d'atteindre par cet article. 
Conditions générales de l'application de l'herméneutique en sciences de l'information

\section{Précisions terminologiques}

Nées au cours de la Seconde Guerre mondiale (Lor 1992), les sciences de l'information - nous l'avons dit déjà - sont un regroupement de disciplines plus ou moins définies qui ont pris pour objet l'information consignée, c'est-à-dire «la connaissance inscrite sous une forme écrite (imprimée ou numérisée), orale ou audiovisuelle» (LeCoadic 1994, 7). En France, l'expression «science de l'information" s'emploie au singulier alors qu'au Québec, le pluriel domine largement dans les écrits. En anglais, on parle parfois de Information Science au singulier, mais la désignation Information Studies semble mieux traduire le niveau actuel du développement de cette discipline qui envisage de plus en plus cette science dans une perspective multidisciplinaire.

Ainsi, l'objet des sciences de l'information demeure l'information au sens large, objet qui varie toutefois en fonction de ceux qui l'étudient. L'archiviste, par exemple, n'étudie pas l'information sous le même angle que le bibliothécaire, et encore moins sous celui de l'informaticien ou du webmestre. Néanmoins, tous s'entendent pour mettre en commun leur expertise, fondant par le fait même une approche commune désignée sous le nom générique de "sciences de l'information ". Quant à la nature de l'information, elle pose certes de nombreux problèmes de fondement théorique, mais ce n'est ni le lieu ni le temps ici de les exposer dans toute leur complexité. Ajoutons simplement que, selon Gilles Deschâtelets $(1990,213)$, le véritable objet de ce regroupement de sciences est le «transfert de l'information d'une source à un utilisateur". Ici la notion de «source » recouvre celle de document, lieu de représentation de la connaissance humaine. Quant à l'information, il s'agit plus précisément de celle qui se manifeste en réponse à un besoin, c'est-à-dire celle à laquelle on accorde une «signification». Le transfert de l'information - notion essentielle à la compré- hension de ce que sont les sciences de l'information - est compris comme une chaîne d'activités communes aux disciplines concernées: évaluation, création; organisation, stockage, repérage et diffusion (ibid., 214). En terminant, il importe d'avoir à l'esprit que cette chaîne d'activités est toujours exécutée dans un souci constant de la part des professionnels: les besoins d'information des utilisateurs. C'est ce qui explique que les sciences de l'information s'avèrent résolument des sciences «humaines», condition obligée de leur appartenance à une discipline distincte.

Les disciplines regroupées dans cette «science » varient en fonction des cultures scientifiques des différents pays. En France, elle regroupe la bibliothéconomie, la muséologie, la documentation et le journalisme tandis que, en Amérique du Nord, elle inclut rarement le journalisme qu'on retrouve surtout dans les départements de communications. Par contre, l'archivistique, discipline beaucoup moins reliée à l'histoire qu'en Europe, en fait toujours partie puisque l'objet de ses interventions est l'information organique et consignée, c'est-à-dire les documents dont la création découle des activités des organisations.

Dans le cadre de ce travail, nous avons recensé quatre applications de l'herméneutique aux sciences de l'information: deux en bibliothéconomie (analyse documentaire et référence), une en gestion de l'information dans Internet et, enfin, une dernière en archivistique (évaluation des archives). Nous verrons ci-après quelles sont les conditions générales de ces applications et à quoi elles correspondent avec exactitude. Mais avant, apportons quelques précisions terminologiques sur ce qu'il sera entendu par "herméneutique» dans les lignes qui suivent.

Tel que le suggère l'étymologie du mot qui fait référence au personnage de Hermès, messager des dieux dans la mythologie grecque, l'herméneutique est une notion qui se rapproche de celles de médiation, de transmission. Elle est donc associée à l'interprétation, à la traduction et, dans un sens plus large, à la compréhension. L'art de comprendre, titre d'un ouvrage du philosophe allemand HansGeorg Gadamer (1982), est sans doute l'énoncé qui décrit le mieux ce qu'est l'herméneutique.

$\mathrm{Au}$ départ, l'herméneutique désigne l'art d'interpréter les textes sacrés ou pro- fanes. Il s'agit donc d'une discipline fort ancienne qui est identifiée tantôt à l'exégèse biblique, tantôt à la philologie. Elle est également comprise comme une réflexion méthodologique sur la pratique de l'interprétation. Ce n'est qu'assez récemment qu'elle a acquis le sens plus large d'une théorie philosophique de l'interprétation (Auroux 1989, 1129). En effet, comme le précise Jean Grondin (1993, xiv), même si l'herméneutique, en tant que science de l'interprétation, remonte aussi loin que l'apparition du terme latin hermeneutica, elle n'en demeure pas moins une philosophie contemporaine qui s'est développée dans le sillage de Wilhelm Dilthey et, surtout, de Edmund Husserl, le père de la phénoménologie (Dupuy 1995, 363).

Si l'herméneutique s'intéresse surtout au texte, son objet s'est aujourd'hui étendu aux œuvres d'art, aux récits mythologiques, aux rêves, à la littérature et même au langage en général (ibid., 362). Dans son étude sur l'universalité de l'herméneutique, Jean Grondin (1993, 2-3) en distingue deux approches. La première, en considérant l'herméneutique comme un art de la compréhension qui propose des règles méthodiques concrètes pour l'interprétation des textes, lui attribue une fonction technique ou normative. La seconde propose plutôt de renoncer à cette tâche, se concentrant davantage sur la réflexion philosophique qui a pour objet la compréhension en tant que phénomène.

Les auteurs en sciences de l'information abordent la question de l'herméneutique en se fondant tant sur la première approche que sur la seconde. Toutefois, une constante se dégage de l'utilisation qu'ils font de l'herméneutique: la majorité d'entre eux perçoivent l'herméneutique comme une solution de rechange aux méthodes quantitatives issues des sciences exactes et appliquées.

\section{Justification du recours à l'herméneutique en sciences de l'information}

Le recours à l'herméneutique en sciences de l'information est un phénomène récent et, qui plus est, encore assez peu courant. En fait, dans notre revue de littérature, nous n'avons relevé que neuf titres qui portaient en totalité ou en partie sur l'herméneutique. De ces titres, un est un compte rendu de lecture (Brown 
1991b), un autre fait état de la question sur l'utilisation des méthodes qualitatives en recherche (Horn 1998); si nous retranchons les titres précédents de l'ensemble, il ne reste plus que sept titres publiés durant les dix dernières années. Cela nous autorise à conclure que l'herméneutique est un mouvement embryonnaire, voire marginal, en sciences de l'information. L'extension de son utilisation aux sciences de l'information témoigne toutefois d'une préoccupation commune à plusieurs auteurs: l'énoncé d'un nouveau fondement épistémologique en sciences de l'information. C'est dans ces conditions que la plupart des auteurs tentent d'appliquer l'herméneutique à leur objet de recherche.

Cette préoccupation est d'abord partagée par Daniel Benediktsson (1989) qui cherche à démontrer la valeur d'une recherche alternative en sciences de l'information, plus particulièrement la valeur des méthodes non empiriques, qualitatives, voire philosophiques. Depuis trop longtemps, estime-t-il, les méthodes empiroquantitatives ont dominé le domaine des sciences de l'information, méthodes qui ont soutenu des recherches dont les valeurs pratiques sont loin d'être évidentes (Benediktsson 1989, 202). Selon lui, l'herméneutique comme théorie de l'interprétation est issue d'une tradition qui remonte assez loin dans le passé: elle permet d'offrir l'autre côté de la médaille. De plus, elle est profondément reliée aux sciences humaines auxquelles se rattachent dorénavant les sciences de l'information. Ainsi, par son article, Benediktsson (1989) prétend faire la lumière sur cette «méthode» afin qu'elle constitue le terrain sur lequel s'élaboreraient d'éventuelles recherches en sciences de l'information. L'auteur croit que l'herméneutique, couplée aux nouvelles technologies, pourrait révolutionner la pratique des sciences de l'information. II compte orienter sa recherche en clarifiant les points suivants: la base philosophique de l'herméneutique, sa pertinence pour les sciences sociales, ses applications possibles aux sciences de l'information.

De son côté, Yvar Hoel (1992) déplore la conception «scientiste» qui domine largement les approches des auteurs en sciences de l'information. Ceux-ci, affirme-t-il, considèrent que les méthodes scientifiques quantitatives doivent s'appliquer aux sciences de l'information, considération dont le fondement trouve son origine dans le développement de la philosophie analytique. Toutefois, il rappelle qu'il existe d'autres philosophies, d'autres méthodes. Et c'est le cas de l'herméneutique (ibid., 73). À l'instar d'autres auteurs (Benediktsson 1989; Budd 1995), Hoel distingue au moins deux approches en herméneutique: l'herméneutique classique, dont les représentants les plus connus sont Schleiermacher (1768-1834) et Dilthey (1833-1911), et l'herméneutique philosophique, soit celle de Hans-Georg Gadamer (1996). Sans opérer une sélection arrêtée de l'une ou de l'autre de ces approches, il estime que le champs qui est plus susceptible d'appliquer l'herméneutique est compris dans ce qu'on appelle les études d'utilisateurs, c'est-à-dire l'identification des besoins d'information des utilisateurs de ressources documentaires (Hoel 1992, 79).

Quant à John Budd (1995), il voit dans l'herméneutique un fondement épistémologique aux sciences de l'information. Pour celui-ci, l'herméneutique n'est ni une méthode ni une problématique nouvelle: «It is a description of a mental state and the public expression of that state" (Budd 1995, 304). Étrangement, l'auteur résume le cheminement herméneutique par Husserl, Heidegger, Ricoeur et Derrida sans qu'il soit question de Gadamer. C'est sans doute parce qu'il considère avant tout l'herméneutique comme étroitement associée à la phénoménologie (ibid., 304-312). Quoi qu'il en soit, d'après Budd (1995), c'est surtout au processus de référence, de communication de l'information, que l'herméneutique pourrait s'appliquer. À la fin de son article, il souligne aussi d'autres applications possibles, notamment en repérage de l'information (indexation), déjà identifiées par Benediktsson (1989).

Parmi les auteurs qui ont rédigé des textes complets sur l'herméneutique appliquée aux sciences de l'information, deux passent sous silence les justifications méthodologiques ou épistémogiques de cet emprunt. II s'agit de Bruno Richardot (1996) et de V.M. Doland (1988). Le premier souhaite simplement asseoir sa «professionnalité» sur une pensée moins techniciste, moins routinière tandis que le second utilise surtout l'herméneutique pour qualifier une caractéristique des documents structurés en hypertexte.

En terminant, il convient de signaler que l'archiviste Richard Brown (1991a) considère l'herméneutique comme le fon- dement possible d'une pratique bien délimitée aux Archives nationales du Canada (ANC) : la stratégie d'acquisition des archives. En ce sens, le recours à l'herméneutique se justifie par le fait qu'elle vient, sur le plan théorique, renforcer une pratique qui, aux dires mêmes de l'auteur, manque de substance et de rigueur méthodologique. Ainsi, pour Brown (ibid., 35), le recours à l'herméneutique permet de transformer cette pratique en un discours cohérent et significatif de l'acquisition des archives et, par le fait même, de leur évaluation.

Pour les auteurs en sciences de l'information, le recours à l'herméneutique traduit donc des préoccupations d'ordre méthodologique susceptibles de trouver appui dans un nouveau fondement épistémologique. Dans les pages qui suivent, nous verront sous quelles formes ces auteurs envisagent l'application de l'herméneutique à leurs champs de connaissances.

\section{Applications de I'herméneutique en sciences de l'information}

\section{Herméneutique et bibliothéconomie}

Parmi les articles consacrés à l'application de l'herméneutique à la bibliothéconomie, nous n'avons recensé que deux documents portant sur des champs spécifiques de cette pratique professionnelle, soit celui de Bruno Richardot (1996) en analyse documentaire et celui de John Cohen (1993) en communication de l'information (référence). Bien qu'il utilise les trois quarts de son texte pour bien cerner la relation entre herméneutique et sciences de l'information, Daniel Benediktsson (1989) identifie ces deux champs comme applications privilégiées de l'herméneutique. La description qu'il fait de ces applications demeure toutefois assez sommaire. Quant aux articles de John Budd (1995) et de Ivar Hoel (1992), d'un intérêt essentiellement épistémologique, ils ne décrivent aucune application pratique de l'herméneutique à la bibliothéconomie. C'est pourquoi nous ne nous contentons que de les signaler. 


\section{Analyse documentaire (bibliographie)}

En bibliothéconomie, la bibliographie s'inscrit dans un domaine compris dans ce qu'on appelle "l'analyse documentaire", domaine qui peut se définir comme une technique qui a pour but "de dériver d'un document un ensemble de mots qui puissent servir à le représenter de façon condensée" (Le Coadic 1994, 72). Le produit de cette analyse prend généralement la forme d'une bibliographie thématique dont les notices sont repérées à l'aide de différents types d'index. En simplifiant sans doute à l'extrême, on peut dire que l'analyse documentaire consiste à offrir une représentation formelle du contenu d'un document, le niveau de profondeur de cette représentation dépendant, d'une part, des besoins documentaires des utilisateurs et, d'autre part, du type de document qui fait l'objet de l'analyse. Par exemple, on n'analyse pas une photographie comme un article de périodique, et encore moins comme une monographie. De même, le travail d'analyse d'un document n'est pas le même dans un centre de documentation scientifique que celui fait dans une bibliothèque publique. Bref, la profondeur de l'analyse dépend des utilisateurs, de leurs besoins, du type des documents ainsi que - inutile de le taire - du budget dont on dispose pour le faire. Signalons que le terme de "bibliographe ", encore assez usité en France, a une connotation plutôt vieillie au Québec. On lui préfère plutôt le terme d'indexeur, voire d'analyste.

Parmi les textes que nous avons consultés, celui de Bruno Richardot (1996) est le seul qui porte spécifiquement sur l'analyse documentaire. Son article se présente comme une réflexion sur la bibliographie en tant que pratique documentaire, réflexion qui s'inscrit dans un projet de dépassement de cette pratique. Après avoir défini en détail les pratiques bibliographiques - signalétiques et analytiques -, Richardot précise leur rôle en développant le concept de "référence» comme "prise de lien ". "Référencer, pour le documentaliste bibliographe, c'est glisser un court texte (une "notice") entre un à-lire concret et un vouloir-lire supposé, discrètement, avec la transparence maximale, afin de ne pas entraver l'établissement de la liaison, la prise de lien". Pour mesurer la qualité d'une référence, l'auteur introduit deux critères: la conformité objective, soit la fidélité de la description, efficace pour l'identification du référencé, et la lisibilité, c'est-à-dire "la référence comme texte donné à lire" (ibid., 10).

Plus loin, Richardot s'arrête sur ce qu'implique la bibliographie analytique d'auteur comme pratique documentaire, rappelant que l'œuvre d'un auteur ne se réduit pas à une indication bibliographique. Par contre, la notice bibliographique, elle, constitue une double réduction: celle de l'œuvre en document, celle de ce dernier en une série de caractéristiques d'identification. En fait, en élaborant une bibliographie, le documentaliste déconstruit l'œuvre, "substituant à une cohérence d'auteur une atomisation d'informateur" et, ce faisant, celui-ci produit un sens qui serait celui de la désignation, «le pointage même d'un sens rendu inaccessible par l'atomisation" (ibid., 10-11).

Afin de dépasser ce simple pointage du sens, l'auteur propose d'enrichir la pratique documentaire par l'exégèse bibliographique. Cette exégèse veut être une œuvre à part entière qui se finalise dans une invitation à lire l'œuvre de l'auteur. Richardot la pense comme l'élaboration d'une méthodologie d'herméneutique documentaire. En effet, la bibliographie n'ajoute pas un sens nouveau au texteobjet: elle ne rend compte que du contenu. Dans l'exégèse, c'est la complexion, l'ensemble de l'œuvre interprétée qui détermine l'écriture documentaire: "II ne s'agit plus seulement d'informer sur mais, plus globalement, de transformer, dans un travail en plusieurs temps de compréhension - décontextualisation - recontextualisation» (ibid., 11).

Puis l'auteur revient aux concepts de référence et de sens. Si la référence bibliographique est une "prise de lien ", la référence herméneutique va plutôt permettre de rendre habitable le monde déployé par le texte. Avant d'aborder de plain-pied la question de l'herméneutique, l'auteur effectue un détour par la critique littéraire et, pour ce faire, il résume les hypothèses de Maurice Blanchot sur la parole critique versus la parole créatrice. Pour l'auteur, l'herméneutique dépasse la critique littéraire en ce qu'elle travaille plus à la lisibilité du monde qui déploie le texte qu'à expliciter les intentions de l'auteur. Cette herméneutique-là est définie par l'auteur comme une "pratique méthodique de l'interprétation" (ibid., 13). Interpréter, c'est s'approprier le monde déployé dans un texte. Autrement dit, c'est le comprendre. En cela, Richardot renvoie aux travaux de Paul Ricoeur (1986). Poursuivant dans la même veine, il postule que toute herméneutique procède par soupçon de sens: "Le langage ne dit pas exactement ce qu'il dit. Le sens qu'on saisit, et qui est immédiatement manifesté, n'est peut-être en réalité qu'un moindre sens, qui protège, resserre, et malgré tout transmet un autre sens; celui-ci étant à la fois le sens le plus fort, et le sens d'en dessous" (Richardot 1996, 13-14).

Ensuite, Richardot propose une série d'étapes conduisant à l'exégèse bibliographique. Ces étapes se résument à l'élucidation de signification, la "contextualisation " des références convoquées dans le texte interprété et la convocation des références non explicitement convoquées dans le texte interprété. II s'agit donc, dans un premier temps, de restaurer le texte et "l'intertexte" et, dans un second temps, d'exposer au-devant du texte interprété le projet de monde qu'il propose. En cela, l'auteur s'appuie sur la pensée de Paul Ricoeur, philosophe français, cité à quelques reprises par l'auteur.

Bruno Richardot insiste plus loin sur le fait que, s'il fait appel à la philosophie, c'est qu'il souhaite asseoir sa «professionnalité " sur une pensée vive et vivifiante, c'est-à-dire productrice de sens. C'est aussi par refus obstiné de la double réduction, techniciste et servile, qu'on impose trop souvent à la fonction documentaire. Mais une herméneutique documentaire est-elle possible? Peut-être... et, pour le démontrer, Richardot décrit un exemple d'exégèse bibliographique d'auteur, exemple qui nous semble trop fragmentaire pour convaincre. La critique du travail bibliographique qu'il fait conduit à la lecture de l'œuvre, comme " ouverture de l'enclos" (ibid., 15). Est-ce le travail du documentaliste, du bibliographe, de lire les œuvres qu'il recense? Nous ne le croyons pas... sinon l'analyste documentaire ne suffirait jamais à la tâche. Mais dans la mesure où celui-ci doit extraire l'essentiel d'une œuvre pour y apposer un indice classificatoire, voire des descripteurs susceptibles de permettre son repérage, et même parfois en faire un résumé représentatif, il est clair que la dimension herméneutique de cette pratique est indéniable.

Benediktsson a très bien compris cette dimension herméneutique de l'analyse documentaire qui consiste à fournir 
une représentation bibliographique d'une $œ u v r e$. Pour lui, les documents et leur représentation sont des symboles et des systèmes de signes qui ont besoin d'être interprétés en fonction d'une herméneutique, notamment celle d'Emilio Betti. En effet, il semble que l'herméneutique de Betti soit intéressante pour le documentaliste pour la recherche documentaire et pour la satisfaction des besoins des utilisateurs. II en va de même pour les mécanismes d'accès à l'information. En conclusion, l'auteur estime que: «Betti's major contribution, and at the same time a most important conclusion for LIS [Library and Information Studies], is that objective knowledge of expressions of meaning is possible, not only in the sphere of value interpretation, but also in all areas where we are confronted by meaningful forms". (Benediktsson 1989, 214).

Là où Benediktsson rejoint Richardot, c'est lorsqu'il estime que la philosophie de Paul Ricœur peut s'avérer utile à l'analyse documentaire. Pour Benediktsson, le grand mérite de Ricoeur est de reconnaître que toutes les écoles ont quelque chose d'unique à offrir et que ces choses ne sont pas mutuellement exclusives. «Particularly the Geisteswissenschaften and, of course, LIS should feel the benefit of a mediated approach since there are a number of practical disciplines within that area in which some degree of flexibility is desirable» (ibid., 216). L'approche de Ricoeur consiste à concilier herméneutique, phénoménologie et structuralisme, et de cet amalgame résulte sa théorie du texte. La portée de son herméneutique est toutefois plus restreinte: «For him, hermeneutics is only interpretation of texts, which seems to be a long way from Betti's all-inclusive domain of the objectivations of mind» (ibid., 216).

Donc, Ricoeur a intégré la phénoménologie de Husserl et le structuralisme à I'herméneutique. II soutient qu'on ne peut arriver à la compréhension par l'interprétation structurale (à la Saussure), mais il insiste sur l'analyse structurale, particulièrement quand elle rencontre le symbolisme. Ses propos sur l'analyse structurale du langage réaffirment que le langage doit être considéré tant comme un événement que comme un système de signes. En ce qui a trait aux applications possibles de l'herméneutique de Ricoeur aux sciences de l'information, Benediktsson croit que c'est surtout dans le domaine de la représenta- tion bibliographique que l'herméneutique de Ricoeur s'avère pertinente. Toutefois, il estime que, en dépit de son intérêt, l'ontologie de Ricoeur n'a pas vraiment d'incidences pratiques en sciences de l'information: "LIS should neither be concerned with ontological questions nor with problems of ontological implications, because it lies both beyond its needs and competency» (ibid., 218). Ainsi la question de l'être (óntologie) intéresse peu Benediktsson, ce dernier préférant plutôt les possibilités qu'offre la représentation bibliographique comme résultat de l'interprétation.

Un peu plus loin dans son article, Benediktsson $(1989,227)$ confirme que l'analyse documentaire, notamment dans son aspect « repérage de l'information », constitue un des deux domaines d'application de l'herméneutique en sciences de l'information. Compte tenu qu'il a été publié bien avant celui de Girardot, l'article de Benediktsson fait figure de précurseur et sa compréhension de l'herméneutique s'avère de loin supérieure à celle de la plupart des auteurs en sciences de l'information.

\section{Communication de l'information (référence)}

Le second domaine d'application de l'herméneutique à la bibliothéconomie que Benediktsson identifie est celui de la référence, plus particulièrement de la question de référence. La référence, que d'aucuns appellent la communication de l'information, suppose une interaction entre le bibliothécaire et l'utilisateur, interaction au cours de laquelle le bibliothécaire doit comprendre la demande de l'utilisateur s'il souhaite y répondre adéquatement. Benediktsson, toutefois, ne fait qu'identifier ce domaine d'application, sans apporter davantage de précision.

Le mérite de consacrer un texte entier à ce domaine d'application revient à John Cohen (1993), un auteur australien qui s'est intéressé à la question de référence envisagée sous l'angle herméneutique. Même s'il constate l'absence quasi totale de sources en sciences de l'information ayant recours à la philosophie herméneutique, il est convaincu que celle-ci est susceptible d'apporter un éclairage nouveau à la question de référence. Par rapport à cette dernière, il souligne toute l'importance qu'elle revêt en bibliothéconomie; la référence étant considérée comme le pi- vot autour duquel tournent toutes les activités d'une bibliothèque (Cohen 1993, 183). II juge d'ailleurs assez étonnant que si peu de chercheurs s'intéressent au sens et à la compréhension de la question de référence, alors que tout le monde connaît le processus de l'émetteur (client) et du récepteur (bibliothécaire) en communication de l'information. En ce qui a trait à l'herméneutique, théorie de l'interprétation qui tire son origine du mythe grec d'Hermès, messager des dieux, l'auteur énonce l'allégorie suivante: «Now it is possible that you might suddenly make a connection between the library and its clients and suggest that Mt Olympus is based on home territory and the reference librarian is really shod with winged shoes" (Ibid., 183).

L'auteur rappelle ensuite le mode de la question de référence élaboré par Robert Taylor en 1965. Ainsi, selon ce dernier, les besoins d'information s'expriment par les étapes suivantes: le besoin est d'abord viscéral (mais non exprimé); il est conscient; il est formalisé ; enfin, il se transforme en compromis puisqu'il se conforme à la question présentée par le système d'information. Une tel processus de la question de référence ouvre de nouvelles perspectives. À l'ère de la société poststructuraliste, on ne peut plus penser que la vie puisse se mesurer avec une cuillère à thé. Des philosophies, comme la théorie de la déconstruction de Jacques Derrida ont ébranlé les fondations de ce qu'on croyait mesurable, quantifiable. La question de référence s'insère maintenant dans une structure qu'il convient d'appréhender. Cette structure est la suivante:

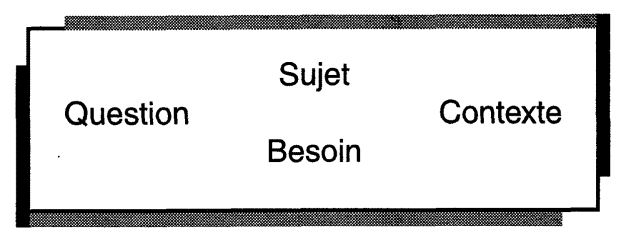

Ainsi: «The question takes on meaning because of the particular structure at the same time the structure does not exist without the meaning or content (ibid., 184). La question de référence ne peut être réduite en éléments distincts. Loin de se réduire à un ensemble de points statiques, elle se présente comme un organisme vivant ayant son propre mouvement qui peut se transférer d'un contexte à un autre, "... it is by the tongue that we hear because listening and speaking are simultaneous acts and are not mutually 
exclusive» (ibid., 184). Ensuite, l'auteur rappelle que les sciences de l'information sont familiarisées avec l'esprit de système - celui de Pavlov (stimulis-réponse), de Skinner, de Shannon et Weaver (théorie de l'information). Toutefois, il insiste sur le fait que, appliqués à la question de référence, les systèmes ont pour effet de briser la dynamique spontanée de la question. Le concept de structure est fondamental dans ce texte. La forme verbale peut être la même pour plusieurs personnes, mais la structure dans laquelle elle s'insère peut différer d'un individu à l'autre.

L'auteur aborde ensuite la question de la non-stabilité du langage. La structure du langage est telle que le sens provient de nous, pas des mots. Il fait ainsi allusion à Ferdinand de Saussure, puis revient à la philosophie de Derrida sur le sens de la question: "Words have no true absolute meanings», et plus loin: «The verbal interaction which takes place between the librarian and the client is an indication of how difficult it is to be certain that the appropriate construct has been grasped" (ibid., 187-188).

En conclusion, les points suivants méritent d'être soulevés:

- La question de référence est loin d'être une simple interaction verbale entre deux individus;

- II s'agit plutôt d'un jeu: «What we have is a constant interplay of dynamic structures that can never be fully grasped" (Cohen 1994, 189);

- Le bibliothécaire de référence ne peut que percevoir approximativement le sens qui se dégage de cette structure interactive;

- La réponse appropriée est celle qui construit un sens aux yeux du client.

Bien qu'il présente de l'intérêt à plus d'un titre, cet article est l'exemple typique de l'utilisation d'un concept — l'herméneutique - comme s'il s'agissait d'un courant, d'une mode. En fait, il s'agit d'un abus de langage. En effet, outre Jacques Derrida qui, d'ailleurs, n'est pas nécessairement un «auteur herméneutique», on ne fait aucune allusion aux philosophes reconnus dans ce domaine, notamment Paul Ricoeur et Hans-Georg Gadamer. II est vrai que la question de référence constitue un problème complexe, mais le recours à l'herméneutique est loin d'être justifié. Du moins, l'auteur n'arrive pas vraiment à vendre l'idée que le recours à l'herméneu- tique pourrait constituer un apport non négligeable à la théorie de la référence en bibliothéconomie. II faut donc revoir cette question pour tenter de mesurer la pertinence de cette application.

\section{Gestion de l'information dans Internet}

La gestion de l'information dans le réseau des réseaux (Internet) constitue un champ d'expertise qu'accaparent de plus en plus les bibliothécaires, lesquels se transforment pour l'occasion en «webmestres ». Qu'elle soit virtuelle ou non, en format HTML ou en bit, l'information circule de plus en plus rapidement et, surtout, sa croissance est plus qu'exponentielle. Un simple clic de souris lui permet d'accéder à des encyclopédies en ligne, à des banques de données, à des périodiques électroniques, etc. Néanmoins, un seul auteur s'est interrogé sur la dimension interprétative des documents accessibles par Internet. Et son interrogation porte sur l'hypertexte comme mode d'organisation des informations dans un document.

\section{Documents structurés en hypertexte}

En sciences de l'information, l'hypertexte est un concept qui a émergé au début des années 1970 en tant que type nouveau de documents électroniques. Selon LeCoadic (1994, 66), il s'agit d'un «véhicule informatique d'une information non linéaire, résultat du démantèlement par le médium ordinateur de l'organisation strictement séquentielle du médium papier ». Ce qui distingue l'hypertexte des autres types de documents, c'est sa structure associative qui reproduit d'assez près la structure de la mémoire humaine et «peut devenir son supplément intime et élargi» (ibid., 68).

On n'a qu'à naviguer dans le réseau des réseaux pour constater l'immense popularité des documents structurés en hypertexte... et de parcourir la littérature pour se rendre compte qu'ils font assez peu l'objet d'une réflexion critique. Une exception toutefois: dans un article plus très récent tiré d'une conférence sur la gestion de l'information en ligne, V. M. Doland (1988) s'est interrogé sur les fondements de l'hypertexte en relation avec la transmis- sion des connaissances par le moyen des bases de données académiques. En fait, l'auteur se demande quel pourrait être l'impact interprétatif de l'organisation massive d'information structurée en documents hypertextes. On aura déjà compris que c'est cet «impact interprétatif » qui renvoie à l'herméneutique.

Doland constate que certains auteurs utilisent la notion de «rhétorique» pour qualifier le caractère «orienté » de l'hypertexte. À cet effet, il adresse le commentaire suivant: « We must continually remind ourselves of the way in which a hypertext systeme is an interpretive act. That is to say, hypertext has its own meaning and impresses its own meaning upon texts; the rhetoric of hypertext must be acknowledged" (Doland 1988, 76). Cela revient à dire que la structure même des documents en hypertexte relève du discours et que, à ce titre, elle ne saurait être exempte d'interprétation.

Les propos de l'auteur s'orientent ensuite vers la question du paradigme au sein duquel un «gardien» balise l'émergence des données scientifiques en imposant des paramètres à la connaissance. La présence d'un autre choix, représentée ici par l'herméneutique, constitue une opposition à ce consensus paradigmatique. La question posée par l'auteur est celle-ci: "How, in the face of an innovation such as hypertext, can we maintain the balance of alternative knowledgess?» (ibid., 77). Pour l'auteur, l'hypertexte n'est pas simplement une extension de l'écrit : c'est un nouvel outil conceptuel susceptible de faire éclater la tradition académique. En outre, l'hypertexte n'est pas non plus qu'une simple approche de gestion de l'information. Dans la mesure où il établit des liens entre des informations dans un document, «... the system is clearly open to interpretive freighting» (ibid., 77). En fait, l'hypertexte conduit à «énoncer du sens" statement about meaning. Certes, le système n'impose pas à l'utilisateur des «liens», mais pour qui veut comprendre le message, ceux-ci s'imposent d'eux-mêmes. En s'appuyant sur l'exemple d'un site sur l'histoire de la guerre civile américaine, l'auteur indique clairement que le lien en lui-même oriente la compréhension de l'utilisateur. Pourquoi? Tout simplement parce que les liens proposés par le concepteur du document ne sauraient être exhaustifs puisqu'il tait les autres qui sont possibles, ce dont les utilisateurs n'ont pas nécessai- 
rement conscience. De cela, l'auteur conclut: "Thus, without special awareness of the cognitive factors implicit within such massively accessible bodies of knowledge as hypertext might create, a great deal of intellectual intoxication might be expected » (ibid., 77-78). Ainsi, la nature de l'hypertexte, en tant qu'outil informationnel et éducationnel, est particulièrement sensible au danger d'arrêt de la compréhension, c'est-à-dire aux choix prédéterminés par le créateur du document. Tout créateur de document structuré en hypertexte doit être conscient de ce danger pour l'éducation et la recherche.

Quant à l'utilisation pédagogique de l'hypertexte, elle s'oppose à la multiplicité des liens et, partant, de la profondeur de la compréhension du document. En effet, pour des raisons pédagogiques, on limite volontairement le nombre de liens, fixant ainsi la compréhension du document avant qu'il ne devienne trop complexe. À cette troncature de l'information s'ajoute l'apparence d'authenticité du document hypertexte. D'après l'auteur, le fait même de créer des liens donne une impression de vérité dont l'enseignant devrait se méfier. Un troisième facteur s'ajoute au précédent: l'exhaustivité. Le nombre de liens, du seul fait de leur existence, donne l'impression que l'information contenue dans le document est complète. Comme le mentionne l'auteur: "Although the paths may. seem infinite, the linkages are ultimately finite and reflect the interpretive choices of the creator of the document " (ibid., 78). Enfin, dans un document hypertexte, l'étudiant navigue sans nécessairement comprendre la relation entre les diverses parties du document et, en conséquence, n'a pas tendance à questionner les présupposés de l'auteur. «In fact, the act of moving from screeen to screen can encourage superficiality» (ibid. 79). Ainsi, peut-on conclure, l'hypertexte comme médium éducationnel peut aggraver le phénomène de la superficialité de la connaissance.

En ce qui a trait à l'hypertexte comme outil de recherche, l'existence de gigantesques bases de données structurées semble contredire la mise en garde de l'auteur. Toutefois, ces données complexes voilent les effets herméneutiques des hyperliens. Par ailleurs, ces systèmes d'information instaurent une certaine canonicité scientifique. À cet égard, l'auteur questionne la légitimité des « gardiens » des systèmes, et ces questions devront trouver ré- ponses si l'on souhaite assurer la crédibilité scientifique de ces outils. Mais le plus grand danger de l'hypertexte, c'est qu'il voile les présupposés des créateurs de documents. Comme Doland $(1988,79)$ le souligne: «The most serious danger of all is that hypertext systems might perpetuate their creator's unconscious assumptions". Cela rejoint la question de la nature du savoir, nature dont l'auteur fait allusion au début de son article. Pour illustrer son propos, celui-ci donne l'exemple d'un document hypertexte relatif à une étude d'auteur. II démontre alors que le seul fait d'établir un lien à «biographie» indique que celle-ci est importante dans une critique littéraire, contrairement à ce qu'en pensent, par exemple, les formalistes. «That is precisely the point: all connections (i.e. linkages) are critical / ideological statements of value, importance, relationship " (ibid., 81). Cela veut dire qu'il n'est pas possible de créer un ensemble neutre de liens puisque l'acte d'établir des hyperliens fonde en lui-même ces présupposés. Bien sûr, ce n'est pas une raison pour abandonner l'hypertexte comme moyen de vulgarisation scientifique, mais il s'agit simplement de prendre conscience de la façon dont nous utilisons un tel médium.

Mais comment donc, pouvons-nous nous assurer que l'hypertexte devienne une extension de l'esprit humain et non pas un canal par lequel il s'échappe? Pour cela, il faut d'abord prendre conscience qu'il n'y a rien qui ne soit «idéologique » ou, si l'on préfère, subjectif en ce sens que toute chose prend la coloration du sujet qui l'énonce. Puis il faut aussi être conscient de la manière dont une base de données peut définir le paradigme d'un champ du savoir et, autant que possible, offrir plus d'un choix idéologique aux utilisateurs. Pour l'auteur, la prudence s'impose si nous voulons continuer à exercer le contrôle de la production des connaissances. En conclusion: «There is nothing conceptually neutral in the cognitive universe, certainly not hypertext " (ibid., 81). C'est ce que l'auteur appelle la neutralité herméneutique.

Le mérite de Doland réside surtout dans le fait qu'il souligne la dimension hautement herméneutique des documents structurés en hypertexte. Bien que sa réflexion ne soit pas dénuée d'intérêt, elle ne repose pas sur un cadre conceptuel clairement défini. En effet, nulle part dans son texte, Doland ne fait allusion à une école de pensée ou à une autre. Quant à l'application de l'herméneutique aux sciences de l'information, elle s'avère assez limitée. En fait, il s'agit plutôt d'un début de réflexion qui ne demande qu'à être enrichie.

\section{Herméneutique et archivistique}

L'archivistique est une discipline qui a connu un développement spectaculaire depuis le début des années 1980. De science auxiliaire de l'histoire, elle a accédé au rang de discipline autonome, délaissant l'histoire pour s'intégrer aux sciences de l'information (Couture, Ducharme et Rousseau 1988). Récemment, un ouvrage portugais de portée internationale (Silva 1999) l'a décrite comme une discipline dont l'appartenance aux sciences de l'information ne fait plus aucun doute. En fait, on parle de plus en plus maintenant d'une archivistique intégrée, c'est-à-dire d'une discipline qui intègre dans ses interventions les documents d'archives de leur conception / réception jusqu'à leur élimination ou leur versement aux archives historiques.

Dans ses interventions, l'archivistique s'appuie sur un principe fondamental: le principe de respect des fonds. Celuici se définit comme «le principe fondamental selon lequel les archives d'une même provenance ne doivent pas être entremêlées avec celles d'une autre provenance et doivent être conservées selon leur ordre primitif s'il existe» (Couture et Rousseau 1994, 64). À ce principe, on l'aura compris, s'ajoute le concept de fonds d'archives qui représente l'ensemble des archives produites ou reçues par une organisation dans le cours normal de ses activités. Le fonds d'archives est à l'archivistique ce que la monographie est à la bibliothéconomie: une unité de traitement. Quant à l'évaluation, elle représente une des sept fonctions archivistiques avec la création, l'acquisition, la description, la classification, la conservation et la diffusion. Elle se définit par « l'acte de juger des valeurs que présentent les documents d'archives (valeur primaire et valeur secondaire) et de décider des périodes de temps pendant lesquelles ces valeurs s'appliquent. » (Couture 1999, 104). 


\section{Évaluation des archives}

L'évaluation est une fonction qui prend de plus en plus d'importance dans la théorie et la pratique archivistiques. Un nombre constant d'articles, d'études et de monographies aux États-Unis, au Canada et, dans une moindre mesure, en Europe sont publiés chaque année sur ce sujet; en témoigne aussi un article récent qui dénombre 176 documents portant directement sur la question de l'évaluation des archives, et ce, sur une période pourtant assez limitée, de 1980 à 1995 (Ducharme et Couture 1996). Donc, cette fonction suscite de l'intérêt et, tant pour des raisons reliées au contexte de compressions budgétaires dans lequel nous vivons que pour d'autres raisons - beaucoup plus fondées - qui s'inscrivent au cœur même de la mission contemporaine de l'archiviste, elle est devenue peu à peu une préoccupation majeure chez les professionnels de la discipline.

Bien que l'évaluation des archives fasse couler beaucoup d'encre et que, en conséquence, elle constitue un terrain propice à l'élaboration de multiples théories, elle n'en demeure pas moins une préoccupation issue de la pratique quotidienne de nombreux archivistes auxquels on vient demander: "Que conserver, quoi détruire?". Cette question, toute simple en apparence, s'avère porteuse d'enjeux fondamentaux pour l'avenir de la discipline archivistique puisqu'elle est reliée à la constitution même du patrimoine archivistique d'une organisation, voire d'une nation. L'image que l'archiviste projette dans la société, son prestige, peuvent être altérés en fonction de la réponse que ce dernier fournira à cette question et, aux yeux des professionnels comme des décideurs, c'est essentiellement à l'archiviste qu'il revient d'en fournir la réponse.

La réponse, les archivistes tentent de la trouver en cherchant dans diverses directions. Le recours à l'herméneutique représente une de ces recherches qui s'inscrit dans un courant bien spécifique: celui de la macroévaluation.

La macroévaluation réfère à une approche développée par des archivistes des Archives nationales du Canada (ANC) qui considèrent que l'évaluation des archives à partir de leur contenu est une pratique révolue. En effet, la complexité des organisations fédérales, le nombre effarant de documents produits, la diversité des supports sur lesquels l'information est consignée sont autant de facteurs qui rendent inefficace une théorie qui cherche à juger des valeurs de l'information contenue dans les documents. Ainsi, au lieu d'évaluer le résultat d'un processus de gestion qui repose sur des fonctions, on propose plutôt d'évaluer les fonctions elles-mêmes, c'est-à-dire d'évaluer les unités administratives productrices d'archives. Pour ce faire, les ANC ont mis au point une records acquisition strategy, c'est-à-dire une politique d'évaluation des fonds gouvernementaux à partir des producteurs, et non des fonds eux-mêmes. C'est précisément dans ce contexte que Richard Brown (1991a) cherche à développer un fondement théorique à l'acquisition stratégique basée sur.l'évaluation du créateur et des formes que prennent ses activités.

L'approche théorique que propose Brown (1991a) repose sur deux énoncés préliminaires: le premier, que les méthodes traditionnelles d'évaluation et de sélection ne sont plus adaptées à la complexité des archives actuelles; le deuxième, que la complexité est devenue la condition endémique de la configuration de la société et, partant, des documents qu'elle génère. En effet, l'auteur constate que les nombreux liens, codes, références et autres structures entrecroisées dont témoignent les documents d'aujourd'hui risquent de constituer le patrimoine archivistique de demain. Cette nouvelle stratégie d'acquisition, toutefois, n'a pas pour but de remplacer l'approche par provenance, mais plutôt d'appeler à un nouvel examen fondamental des principes sur lesquels sont basés nos décisions d'évaluation en offrant un choix, voire un complément, à l'analyse des documents visant à prolonger les catégories de la signification de la provenance. Mais avant, l'auteur s'arrête sur le concept de stratégie, lequel suggère un plan d'attaque ou la gestion tactique d'une campagne militaire en vue de défaire un ennemi. En évaluation, l'ennemi est double: "I think it is safe to say that both the unguided, unreasoned, haphazard accumulation of documentation, and the stockpilling of records with marginal or no historical value whatsoever, must cease forthwith, lest we become buried under an avalanche of disordered, irrelevant information, or offer to succeeding generations a corpus of records incidental or peripheral to the contemporay-historical discourse of our society» (Brown 1991a, 38-39).

Les ANC recourent à trois moyens pour évaluer les archives. D'abord, on cherche à identifier les sources génériques (fonds d'archives) des documents d'archives potentiellement significatifs en accord avec les champs d'acquisition acquisiton targets - déterminés par les mandats de la politique. Ensuite, on classe ces champs par ordre de priorité d'acquisition. Enfin, on subdivise chacun de ces champs en composantes administratives internes en raison de leur valeur de potentialité historique au niveau du sous-fonds, de la série, etc. C'est ce qu'on appelle le records disposition plan.

Du point de vue théorique, cette stratégie d'acquisition est le synonyme de la macroévaluation, approche qui insiste davantage sur la valeur archivistique des sites de production des documents plutôt que sur la valeur des documents euxmêmes, assignant la primauté au contexte dans lequel les archives sont créées, et non pas à la valeur de l'information contenue dans les documents. Pour Brown (1991a, 39), il ne fait aucun doute que celle-ci constitue une approche holistique des archives gouvernementales. De plus, elle appelle à l'élaboration de principes d'une forme naissante d'herméneutique des archives qui trouve son application dans le contexte au sein duquel les archives sont créées et encodées.

L'auteur conçoit l'herméneutique comme la théorie de l'interprétation, comme la philosophie de la compréhension, laquelle postule que, pour comprendre le comportement humain, on doit interpréter sa signification. "In other words, to comprehend fully the dimensions of human experience, the interaction context of human affairs assumes a greater degree of importance as a source of historical knowledge than an empirically-based analysis and scientific observation of natural events» (ibid., 39). Cela conduit l'auteur à discréditer la valeur subjective de l'information contenue dans les documents pour se concentrer sur la valeur objective du témoignage dérivant du contexte de création. Bref, il s'agit d'identifier la valeur historique des documents inhérente à leur production, composition, formation et organisation. Par ailleurs, «... the theory advocates a form of deconstruction by reducing the universe of government information to the sources of its primary signification, its administration ethos 
or network of bureaucratic sub-components, so as to establish tiers of archivalhistorical value based on the appraisal of bureaucratic context(s) and records creators" (ibid., 40). L'auteur estime que cette voie permet de faciliter la localisation et l'identification des sources, d'une part, et d'éliminer l'évaluation des documents basée sur des valeurs qui ne sont pas nécessairement conséquentes avec la signification du mandat d'acquisition et de son interprétation, d'autre part.

L'auteur se penche ensuite sur la stratégie d'acquisition telle que pratiquée aux ANC, stratégie qui prescrit une évaluation active et une acquisition planifiée plutôt que la réception passive des documents. Le succès de cette stratégie repose sur des facteurs radicalement différents de l'approche traditionnelle en évaluation des archives. Ces facteurs peuvent être la signification et la formation (construction) des fonds d'archives et/ou des créateurs ainsi que notre habileté à évaluer les fonds en entier plutôt que leurs parties constitutives; les critères qui guident l'évaluation des parties du fonds n'étant pas nécessairement applicables au fonds entier au sein d'une structure bureaucratique. Autrement dit: Qu'est-ce qui doit être considéré dans la détermination de la valeur archivistique du créateur? Comment peut-on cibler nos acquisitions en tenant compte de l'interaction entre les différentes structures de l'organisation? Devons-nous accepter le jugement que le gouvernement porte luimême sur les mérites du fonctionnement de ses structures? Pour l'auteur, il est clair que la réponse est non... sinon cela reviendrait à considérer automatiquement que les archives situées dans les instances hiérarchiques supérieures ont davantage de valeur que celles disséminées dans d'autres instances de l'organisation: «Here is the environment of government policy and decision-making, not necessarily the location of its operational application, and certainly not the site of its interaction with society at large" (ibid., 41). Ainsi, la circulation de l'information dans les structures organisationnelles n'est pas toujours configurée comme l'organigramme de l'organisation le laisse croire. C'est pourquoi la notion de «fonction» prend de plus en plus d'importance dans l'évaluation des structures qui abritent les documents de l'organisation.

L'auteur commente ensuite les réflexions de Michel Foucault sur le système et le discours qui le sous-tend: «To translate this hypothesis into archival terminology, records creators are simultaneously the product of structural or systemic evolutionary development, and of a modality of discursive representation embodied in the narrative texts of their recorded dialogue " (ibid., 44). Si l'auteur fait ce parallèle, c'est qu'il croit qu'une stratégie d'acquisition doit débuter par un modèle d'analyse qui mettrait l'accent sur les interrelations entre les structures, fonctions et processus institutionnels. Ce qui distingue Brown des autres archivistes canadiens, c'est qu'il estime que l'évaluation de ces interrelations réside dans la lecture des documents considérés comme discours corporatif. Bien qu'il soit d'accord avec la méthode des ANC qui considère, en première instance, les sites ciblés de production d'archives signifiantes et non le contenu des documents, il déplore le fait qu'on néglige de développer la théorie impliquée dans cette façon de procéder. En effet, il juge essentiel d'asseoir cette «première étape» (la macroévaluation) sur une assise théorique reposant sur la «lecture" des archives comme textes narratifs, ce qui permettrait de comprendre ce qui est implicite dans la formation, la production, la structure et la rhétorique des documents.

Pour Brown (1991), plusieurs raisons justifient le recours à l'analyse textuelle dans le cadre d'une macroévaluation. D'abord, dans le contexte de la bureaucratie fédérale, on n'est jamais certain de la véritable identité du créateur d'archives. Il estime qu'il est de plus en plus difficile d'identifier la source première, le créateur originel du fonds, ce qui représente une limite assez sérieuse aux prétentions de la macroévaluation. II questionne d'ailleurs tout le processus d'anticipation d'évaluation des fonds d'archives en relation avec leurs sites. En effet, il estime que: « With the first round of appraisal research completed, at least in some cases, I am anticipating that the archival-historical significance of the records will not generally tally with the ranking of the records creators as it is now apprehended" (ibid., 46). Et l'auteur d'invoquer la complexité des structures et fonctions dont certaines sont apparentées au créateur du fonds. Le principe de provenance (respect des fonds), pour tenir compte de cette complexité, devrait être perçu d'une manière différente: «In this case, archival fonds would be defined exclusively by their physiological referen- ces to specific or generic functions and processes of government, rather than by bureaucratic structures of administration rooted in allocated fields of responsability or jurisdictional portfolio» (ibid., 47). II s'agit donc de repenser le concept de record group eu égard à l'organisation des archives gouvernementales.

Toutefois, l'auteur ne croit pas que la réduction de la portée du principe du respect des fonds l'emporte sur les problèmes pratiques et intellectuels du fondement théorique d'une telle approche. SeIon lui, les nouvelles valeurs doivent être déterminées à partir de l'interprétation du contexte bureaucratique (corpus de connaissances des structures et fonctions de l'organisme) plutôt que déduites en vertu d'une théorie archivistique préméditée, c'est-à-dire «... in the manner that the archival significance of documentation is conventionally assessed in relation to certain categories of value and their subjective connection to preselected systems or structures" (ibid., 47-48). En effet, au principe de provenance, l'auteur inclut l'identification du créateur ou du contexte de création basée sur les connaissances relatives aux fonctions dudit créateur. Ce faisant, il écrit que nous devons apprendre à «lire» les archives gouvernementales comme un discours narratif sur les activités de l'État, comme un témoignage de ses fonctions et opérations. Loin de proposer un retour aux documents, Brown estime que: «... I am advocating a critical examination of homogeneities of texts (records) sufficient to make decisions concerning the identity of records creators (or fonds), and facilitate their rankin in on order of collecting priority» (ibid., 48). Et il pose les questions suivantes: Cela peut-il se faire dans le cadre de la macroévaluation? Suffit-il de renforcer le principe de provenance pour que ça fonctionne?

La réponse à ces questions se trouve dans l'application de l'herméneutique à l'évaluation des archives. Comme le précise Brown «... the philosophy of hermeneutic disposes of the interpretation of information in favour of the interpretation of the site or environment in which information is created and encoded". II s'agit donc de porter attention à la signification implicite dans les différentes actions relatives à la production documentaire plutôt qu'à leurs résultats, c'est-à-dire le contenu. Ainsi, une approche herméneutique concerne surtout la valeur interprétative 
du contexte de création. «In others words, the discursive substance or "narrativity» of texts would be analysed and appraised solely in view of establishing the identity and activity of records creators" (ibid., 48).

En ce qui a trait à la philosophie herméneutique, l'auteur n'a pas la naïveté de croire à la convergence des différentes écoles de pensée. Pour lui, l'herméneutique présente de l'intérêt dans la mesure où elle apporte une consistance intellectuelle au rejet de la signification subjective des textes. Elle peut aussi conduire à une conception de la compréhension (évaluation) qui met l'accent sur l'interprétation du contexte de création des archives, sur le sens (valeur) des archives à travers la connaissance du créateur.

L'auteur distingue deux domaines d'application de l'herméneutique à l'archivistique. Le premier est l'interprétation du contexte en tant que fondement de la représentation historique, ce qui a pour conséquence d'inviter l'archiviste à localiser et à identifier les créateurs de documents. Le second domaine, c'est la réduction de l'information consignée à sa principale source de création, «... or to deconstruct from the organic-documentary whole the genetic texts or discourses concerning the contextual environment of their formation and production" (ibid., 49). Ainsi l'auteur voit le contexte comme étant inscrit dans le texte et le discours, et le créateur comme reconstruit à partir des documents. Dans le cadre d'une stratégie d'acquisition se pose la question suivante: Quelle est la nature du texte-contexte ou de la relation entre les documents et leurs créateurs?

Après avoir situé la notion de conscience historique dans le champ de l'herméneutique, l'auteur rappelle que les ANC, avec leur proposition de records acquisition strategy, ont remplacé l'évaluation des documents par celle de leurs créateurs, l'évaluation du texte par celle du contexte. "Nevertheless, we have not yet engaged the critical question posed by the relationship of context and text, or records creator and records" (ibid., 50). En effet, l'auteur estime qu'on préfère noyer les questions fondamentales dans les procédures et reporter les problèmes de justification de la sélection et de l'élimination à plus tard.

Pour Brown (1991), il est clair qu'on doit effectuer une relecture des archives gouvernementales comme témoignage du contexte bureaucratique. II aborde alors la question du analytic format dont la clé réside dans l'acceptation de la notion de document comme sources narratives du contexte, d'une part, et dans la réalisation de la «narrativité » de leur formation, classement, organisation et inscription dans le discours comme sources des fonds, d'autre part. Quand l'auteur réfère au discours narratif, c'est en conjonction avec l'idée de fonds archivistique. Par la "narrativité » du discours, il entend « an ontological assembly of texts, a metacode of shared textual reality, or a cohesion, convergence or unity of narrative (a fonds of records) in which an implicit understanding of a particular functional activity or structural formation is represented (context in text)» (ibid., 50). En fait, cette conception du discours narratif comme fonds d'archives offre des perspectives nouvelles en matière d'interprétation de la provenance. "In effect, the notion of discourses as fonds presumes a hermeneutic moment, a setting of action in context; a reflexive relation between social structure and situated action the intelligibility of which is wholly determined by a contextdependence of meaning inscribed in the narrativity of texts and discourse" (ibid., 51). Appliquée aux archives, cela suppose l'identification du créateur à partir des messages implicites encodés dans les documents, principalement les connections démontrées par l'implication de la fonction et du processus.

L'auteur rappelle qu'il est en partie d'accord avec la stratégie d'acquisition des ANC qui propose de concentrer l'effort d'évaluation sur l'analyse fonctionnelle des structures administratives de l'organisation (ibid., 51). Toutefois, il invite les archivistes à reconnaître le besoin d'étudier la formation du discours narratif implicite dans certains documents en tant que sources du contexte pour les créateurs. Selon lui, il ne convient pas d'encourager la lecture des documents dans le but de les évaluer en fonction de valeurs archivistiques ou de conclure à la signification archivistique des documents en tant que sources potentielles pour l'histoire. II faut plutôt développer notre connaissance du créateur d'archives et de ses relations avec les structures bureaucratiques de l'organisation fédérale.

L'auteur revient sur l'approche de la macroévaluation en décrivant ses deux principales phases. La première consiste à identifier des cibles d'acquisition (acquisition targets) tandis que la seconde, qui va beaucoup plus loin, vise à confirmer les décisions initiales en établissant un plan stratégique, c'est-à-dire en établissant des priorités. "It is precisely in this research phase that the quasi-hermeneutic concept of context-dependent fonds and the critical reading of records as narrative discourse can have a significant impact, principally by alerting archivists to homogeneities of texts and sub-texts (discourses) which nominate alternative functional and structural loci of bureaucratic activity not generally accounted for by the official rhetoric and representations of government » (ibid., 52). En apprenant à lire les archives comme sources du discours (contexte) plutôt que comme sources de valeurs (information), l'archiviste peut accroître sa connaissance de la structure bureaucratique.

Comme nous l'avons fait pour Doland (1988), reconnaissons à Richard Brown (1991a) le mérite d'être le seul archiviste, à ce jour, à tenter d'appliquer l'herméneutique à l'archivistique. II le fait, certes, d'une manière encore assez confuse, mais sa perception du fonds d'archives comme discours narratif susceptible d'apporter une source d'information privilégiée sur le producteur du fonds, c'est-à-dire sur l'unité administrative d'une organisation, ne manque pas d'intérêt, surtout dans le cadre d'une approche - la macroévaluation - qui préconise l'évaluation des fonctions et structures administratives plutôt que des archives elles-mêmes. Néanmoins, la question de l'herméneutique en archivistique nécessite une recherche beaucoup plus approfondie, recherche qui permettrait peut-être de «penser » davantage le fonds d'archives comme «représentation" de l'organisation. Cette recherche, toutefois, reste à faire, mais Richard Brown pose un premier pas, traçant par la même occasion un chemin prometteur.

En terminant, avouons notre surprise en constatant que les sources «herméneutiques » de cet archiviste canadien-anglais sont toutes françaises. En effet, les seuls philosophes que Brown se permet de citer sont Paul Ricoeur, Michel Foucault et Jacques Derrida. 


\section{Conclusion}

Au terme de cet état de la question, une constatation s'impose d'emblée: l'application de l'herméneutique aux sciences de l'information ne représente qu'un courant embryonnaire, marginal, qui cherche encore vers quelle direction il pourrait bien se diriger. En fait, le recours à l'herméneutique est motivé par deux raisons complémentaires. En premier lieu, l'herméneutique vient combler un vide épistémologique, voire méthodologique, en sciences de l'information et, ce faisant, elle cherche à constituer une approche différente des méthodes quantitatives seules autorisées, jusqu'à tout récemment, à être qualifiées de «scientifiques» (Benediktsson 1989, Budd 1995, Hoel 1992). Dans un second lieu, l'herméneutique est utilisée pour renforcer une pratique existante, voire pour la dépasser en proposant un autre savoirfaire (Richardot 1996, Brown 1991a). Dans l'un comme dans l'autre cas, nous croyons que cette recherche d'application s'avère pleinement justifiée, et ce, pour la simple raison que partout où il $y$ a texte et discours (et en sciences de l'information, il y en a toujours), une dimension interprétative est présente, dimension qui, par le fait même, nécessite une «compréhension».

Ainsi, la pertinence de l'application de l'herméneutique aux sciences de l'information est, à nos yeux, établie. Toutefois, les auteurs en sciences de l'information ne doivent pas s'arrêter là: la recherche en ce domaine n'est qu'à ses débuts. En effet, trop d'auteurs connaissent mal les tenants et aboutissants de l'herméneutique, ne percevant encore pas très bien la distinction entre l'herméneutique philosophique (Heidegger, Gadamer), issue de la tradition allemande, et l'herméneutique historique (Dilthey). De plus, on ne comprend pas très bien non plus le rôle prépondérant qu'a joué la phénoménologie, présup- posé véritable à toute philosophie herméneutique selon Benediktsson $(1989,206)$, dans le développement de l'herméneutique. Par ailleurs, certains voient dans l'herméneutique une méthode qualitative utilisée en sciences humaines alors que d'autres s'arrêtent surtout à son potentiel théorique.

En sciences de l'information, l'herméneutique en est encore aux balbutiements. Notre travail de recensement, nous le croyons, l'a démontré sans peine. Nous espérons seulement qu'il donne envie à certains auteurs de poursuivre la recherche.

\section{Sources consultées}

Auroux, Sylvain. 1989. Les notions philosophiques: dictionnaire. Tome 1: Philosophie occidentale: A-L. In Jacob, André (dir.). Encyclopédie philosophique universelle. Paris: PUF, p. 129-1133.

Benediktsson, Daniel. 1989. Hermeneutics: dimensions toward LIS thinking. Library of Information Science Research 11 (1): 201-234

Brown, Richard. 1991a. Records acquisition strategy and its theoretical foundation: the case of a concept of archival hermeneutics. Archivaria 33 (Winter) : 34-56.

$.1991 \mathrm{~b}$. The value of narrativity in the appraisal of historical documents: foundation for a theory of archival hermeneutics. Archivaria 30 (Summer): 152-156.

Budd, John M. 1995. An epistemological foundation for library and information science. Library Quarterly 65 (3) : 295-318.

Cohen, John. 1993. The hermeneutics of the reference question. The Australian Library Journal (August): 182-189.

Couture, Carol et collaborateurs. 1999. Les fonctions de l'archivistique contemporaine. Sainte-Foy: Presses universitaires du Québec.

Couture, Carol, Jacques Ducharme et Jean-Yves Rousseau. 1988. L'archivistique a-t-elle trouvé son identité? Argus 17 (2): 51-60.

Couture, Carol et Jean-Yves Rousseau. 1994. Les fondements de la discipline archivistique. SainteFoy: Presses de l'Université du Québec.

Deschâtelet, Gilles. 1990. L'archivistique et la bibliothéconomie: deux disciplines sœurs dans l'arbre généalogique des sciences de l'information. In
Groupe interdisciplinaire de recherche en archivistique. La place de l'archivistique dans la gestion de l'information: perspectives de recherche, Centre d'archives de Montréal, 2-3 février 1990. Montréal: Université de Montréal, Service des archives, p. 207-230.

Doland, V.M. 1988. The hermeneutics of hypertext. In Online Information 88: $12^{\text {th }}$ International online information meeting, London 6-8 December 1988. Orford: Learned Information, 75-82.

Ducharme, Daniel et Carol Couture. 1996. L'évaluation en archivistique, évolution et tendances: étude bibliographique, 1980-1995. Archives 28 (1): 59-98.

Dupuy, Bernard. 1995. Herméneutique. In Encyclopaedia Universalis, Corpus, vol. 11. Paris, p. 362365.

Gadamer, Hans-Georg. 1982. L'art de comprendre. Trad. de M. Simon. Paris: Aubier Montaigne.

.1996. Vérité et méthode: les grandes lignes d'une herméneutique philosophique. Trad. Par Jean Grondin. Paris: Seuil.

Grondin, Jean. 1993. L'universalité de l'herméneutique. Paris: Presses universitaires de France.

Hoel, Ivar A.L. 1992. Information science and hermeneutics: should information science be interpreted as a historical and humanistic science? In Vakkari, Pertti and Blaise Cronin. Conceptions of library and information science. London: Taylor Graham, p. 69-79.

Horn, Jim. 1998. Qualitative research literature: a bibliographic essay. Library Trends 46 (4): 602 615.

LeCoadic, Yves-François. 1994. La science de l'information. Paris: Presses universitaires de France.

Lor, P.J. 1992. Contempory dimensions of the information problem. Mousaion 10 (1): 3-28.

Richardot, Bruno. 1996. Des pratiques bibliographiques à l'herméneutique documentaire: sens et références en documentation. Documentaliste - Sciences de l'information 33 (1): 9-15.

Ricoeur, Paul. 1986. Du texte à l'action: essais d'herméneutique. Paris: Seuil.

1989. Narrativité, phénoménologie et herméneutique. In Jacob, André (dir.). Encyclopédie philosophique universelle: I. L'univers philosophique. Paris: Presses universitaires de France, p. 63-71.

Silva, Armando Malheiro et al. 1999. Arquivística: Teoria et prática de uma ciência da informação. Porto, $\mathrm{Pt}$ : Edicões Afrontamento. 